\title{
La sélection des idées prometteuses dans le discours collectif d'élèves du primaire
}

The selection of promising ideas within the collective discourse of primary students

\section{La selección de ideas prometedoras en el discurso colectivo de estudiantes de primaria}

\author{
Pier-Ann Boutin, Christine Hamel et Thérèse Laferrière
}

Volume 18, numéro 2, 2015

URI : https://id.erudit.org/iderudit/1036034ar

DOI : https://doi.org/10.7202/1036034ar

Aller au sommaire du numéro

Éditeur(s)

Faculté d'éducation, Université de Sherbrooke

ISSN

1911-8805 (numérique)

Découvrir la revue

Citer cet article

Boutin, P.-A., Hamel, C. \& Laferrière, T. (2015). La sélection des idées prometteuses dans le discours collectif d'élèves du primaire. Nouveaux cahiers de la recherche en éducation, 18(2), 90-114. https://doi.org/10.7202/1036034ar
Résumé de l'article

Cette étude traite principalement des usages d'un outil permettant la sélection d'idées prometteuses (IPROM) dans le discours collectif par des élèves du $2^{\mathrm{e}}$ cycle et $3^{\mathrm{e}}$ cycle du primaire lors de deux projets vécus en collaboration en réseau. Ces projets portaient sur la rédaction d'un texte informatif en français sur le thème du Moyen Âge et la conception d'une balle en science et technologie. Les élèves ont décrit leur usage de l'outil IPROM pour la sélection des idées prometteuses comme étant surtout au bénéfice de l’individuel, mais ils ont aussi mentionné que cet outil les aidait dans l'élaboration d'un discours collectif. De plus, les façons de contribuer au discours sélectionnées comme étant des idées prometteuses sont majoritairement énoncées comme des théories, des idées des élèves à approfondir. L'utilisation de l'outil IPROM en classe permettrait donc aux élèves du primaire d'entrer dans un processus de coélaboration de connaissances. 


\title{
La sélection des idées prometteuses dans le discours collectif d'élèves du primaire
}

\author{
Pier-Ann Boutin \\ Christine Hamel \\ Thérèse Laferrière
}

Université Laval

\section{Résumé}

Cette étude traite principalement des usages d'un outil permettant la sélection d'idées prometteuses (IPROM) dans le discours collectif par des élèves du $2^{\mathrm{e}}$ cycle et $3^{\mathrm{e}}$ cycle du primaire lors de deux projets vécus en collaboration en réseau. Ces projets portaient sur la rédaction d'un texte informatif en français sur le thème du Moyen Âge et la conception d'une balle en science et technologie. Les élèves ont décrit leur usage de l'outil IPROM pour la sélection des idées prometteuses comme étant surtout au bénéfice de l'individuel, mais ils ont aussi mentionné que cet outil les aidait dans l'élaboration d'un discours collectif. De plus, les façons de contribuer au discours sélectionnées comme étant des idées prometteuses sont majoritairement énoncées comme des théories, des idées des élèves à approfondir. L'utilisation de l'outil IPROM en classe permettrait donc aux élèves du primaire d'entrer dans un processus de coélaboration de connaissances.

Mots clés: coélaboration de connaissances, idées prometteuses, discours collectif, élèves, primaire

\section{The selection of promising ideas within the collective discourse of primary students}

\section{Abstract}

This study primarily examines the use of a tool for selecting promising ideas (IPROM) from the collective discourse of cycle 2 and cycle 3 (years 3-6) primary students in the context 
of two projects carried out in a collaborative network setting. The projects involved writing an informative text in French on the topic of the Middle Ages, as well as designing a ball in Science and Technology. The students described their use of IPROM in selecting promising ideas as being mainly helpful on an individual level, but also mentioned that the tool helped them develop their collective discourse. Moreover, the potential ways to contribute to the discourse that were selected as promising ideas were mainly formulated as theories and student ideas to be further developed. The in-class use of IPROM thus appears to enable primary students to engage in a knowledge building process.

Key words: knowledge building, promising ideas, collective discourse, student, primary school

\section{La selección de ideas prometedoras en el discurso colectivo de estudiantes de primaria}

\section{Resumen}

Este estudio examina principalmente el uso de una herramienta que permite la selección de ideas prometedoras (IPROM) del discurso colectivo de los estudiantes del segundo y tercer ciclo (tercero a sexto grado) de primaria en el contexto de dos proyectos llevados a cabo en un entorno de red de colaboración. Estos proyectos trataban de la escritura de un texto informativo en francés sobre el tema de la Edad Media, así como sobre la concepción del diseño de una pelota en Ciencia y Tecnología. Los estudiantes describieron el uso de IPROM para la selección de ideas prometedoras como una herramienta principalmente útil a nivel individual, pero también mencionaron que ésta les ayudó en el desarrollo de un discurso colectivo. Además, las formas posibles para contribuir al discurso que fueron seleccionadas como ideas prometedoras fueron en su mayoría enunciadas como teorías e ideas de los estudiantes a ser profundizadas. La utilización de la herramienta IPROM en clase permitiría entonces a los estudiantes entrar en un proceso de co-elaboración de conocimientos.

Palabras clave: co-elaboración de conocimientos, ideas prometedoras, discurso colectivo, alumnos, primaria 


\section{Problématique}

L'accès de plus en plus élargi aux outils technologiques agit comme un important moteur de la société actuelle (Collins et Halverson, 2009). Dans le monde de l'éducation, les technologies de l'information et de la communication (TIC) offrent aux élèves et aux enseignants de nouvelles possibilités et elles pourraient permettre d'enseigner autrement, mais aussi d'apprendre autrement (Laferrière, 2005). C'est ce que se propose de faire l'initiative de l'École éloignée en réseau (ÉÉR) mise sur pied dès 2002. Mise en place au moment où la connectivité se déployait sur le territoire québécois ${ }^{1}$, les chercheurs de l'ÉÉR ont fait l'hypothèse que la mise en réseau des écoles pourrait enrichir l'environnement éducatif des élèves (Laferrière et Breuleux, 2002). Un dispositif d'innovation combinant la visioconférence, disponible directement dans la classe, et un outil d'écriture (forum électronique de coélaboration de connaissances, Knowledge Forum) a été expérimenté dès ses débuts dans une dizaine d'écoles et elle est toujours présente dans une centaine d'écoles. Dans le cadre de l'ÉÉR, les enseignants sont appelés à utiliser les technologies afin de mettre l'élève au centre de ses apprentissages et encourager le travail collaboratif. L'utilisation de la plate-forme d'écriture devient l'occasion pour l'élève d'interagir, de négocier, de recevoir des rétroactions rapides et d'articuler un discours afin de créer un savoir collectif autour d'un objet d'investigation de façon asynchrone.

La création de connaissances, telle que décrite par Bereiter (2002) et Paavola, Lipponen et Hakkarainen (2004), n'est habituellement pas mise de l'avant dans le milieu scolaire, et ce, même si elle est indispensable dans le développement des compétences du $21^{\text {e }}$ siècle chez les élèves (Partnership for Skillsfor 215t Century, 2008; Scardamalia, Bransford, Kozma, et Quellmalz, 2012). De plus, comme le soutient Papert (2000), les idées sont au centre du travail intellectuel, cependant, elles sont bien souvent écartées du discours en classe. Le travail avec les idées est un des moteurs de l'apprentissage lors des situations de résolution de problèmes complexes et authentiques. Comme l'affirment Chen, Chuy, Resendes, Scardamalia et Bereiter (2011), I'habileté à repérer les idées de valeur est essentielle au travail créatif. Dans une équipe de recherche, par exemple, une nouvelle idée peut être mise de côté par les membres et une autre, jugée plus prometteuse, peut être mise de l'avant. Avec le temps et l'expérience, les individus améliorent leur capacité

L'initiative gouvernementale Villages branchés, qui fut lancée le 18 septembre 2002, visait à soutenir les commissions scolaires, les municipalités et leurs établissements d'enseignement privés ou publics, primaires et secondaires ainsi que des organismes municipaux (bibliothèques publiques) dans leur désir de mettre sur pied un réseau de télécommunication entre les établissements d'une région. Cette politique fut inspirée par le désir de permettre l'accès aux télécommunications à tous les citoyens québécois, dans un désir d'égalité des chances face aux technologies qui devenaient de plus en plus présentes et incontournables. De plus, c'est par cette initiative que celle de l'École éloignée en réseau a été rendue possible, à compter de 2002, dans plusieurs régions éloignées du Québec. Téléaccessible à www.mels.gouv.qc.ca/lancement/villagesbranches/. 
à prendre des risques fructueux, en ce qui a trait au repérage des idées et à la poursuite de celles-ci (Bereiter et Scardamalia, 2003). Afin de préparer les élèves à participer à la société en tant que créateurs de savoir, il importe d'encourager les jeunes à utiliser leur jugement prometteur (Chen et al., 2011; Chen, Resendes, Chuy, Tarchi, Bereiter et Scardamalia, 2012a; Chen, Scardamalia, Resendes, Chuy, et Bereiter, 2012b).

\section{Cadre théorique}

\subsection{La coélaboration de connaissances}

Nous entendons par coélaboration de connaissances le plus haut niveau de construction collective de sens par un groupe, dans le but de se donner et d'approfondir des connaissances collectives sur un objet (question ou problème). Scardamalia et Bereiter (2003) décrivaient la coélaboration de connaissances en ces termes:

Knowledge building may be defined as the production and continual improvement of ideas of value to a community, through means that increase the likelihood that what the community accomplishes will be greater than the sum of individual contributions and part of broader cultural efforts. (p. 1370)

La coélaboration de connaissances se base sur deux prémisses, soit l'élaboration d'un questionnement authentique et l'élaboration collective d'idées originales de la part des élèves (Bereiter et Scardamalia, 2010). Le processus de coélaboration demeure essentiellement le même que l'on soit au début de la scolarisation chez l'enfant ou bien dans une équipe de recherche, car son but est de faire avancer l'état des connaissances de la communauté. Ce processus requiert que les participants prennent une part active dans leurs apprentissages et dans ceux de la communauté à laquelle ils appartiennent (Bereiter et Scardamalia, 2003). Les questions authentiques se constituent souvent en problèmes dont la réponse est une contribution à la connaissance de la communauté et cette réponse n'est pas uniquement de nature factuelle. Les idées originales sont des idées provenant des participants, qui deviennent le produit des interactions de la communauté, de la négociation de sens. La coélaboration de connaissances se veut un engagement des participants à aller au-delà des connaissances acceptées (belief mode) et à approfondir leur compréhension de l'objet investigué (design mode) (Scardamalia et Bereiter, 2003). 


\subsubsection{Les idées prometteuses et le jugement de la communauté}

Le concept d'idée prometteuse désigne les idées qu'une communauté d'apprentissage identifie comme ayant le potentiel de faire avancer le discours collectif dans le but de résoudre un problème complexe et authentique. Le concept d'idée prometteuse vient du principe que toute idée peut être améliorée par la négociation de sens entre les participants (Bereiter et Scardamalia, 2003). Pour Chen, Scardamalia et Bereiter (2015), une idée prometteuse est jugée comme telle par la communauté qui souhaite l'investiguer davantage, la raffiner et faire en sorte que les idées et les connaissances de chacun progressent. La sélection des idées prometteuses se veut un moteur de complexification de l'objet d'investigation et veut agir, en ce sens, sur le processus d'amélioration des idées, comme l'illustre la figure 1. Le problème authentique de départ est élaboré, ensuite viennent les premières idées des élèves sur le problème. La sélection des idées prometteuses se produirait par la suite afin de miser sur certaines idées étant jugées par la communauté comme ayant le potentiel de repousser l'état des connaissances des élèves en regard du problème de départ. De cette sélection, peuvent découler de nouveaux questionnements plus pointus et complexes en ce qui a trait aux idées prometteuses sélectionnées. S'engage ensuite une nouvelle phase d'amélioration des idées et de complexification de l'investigation.

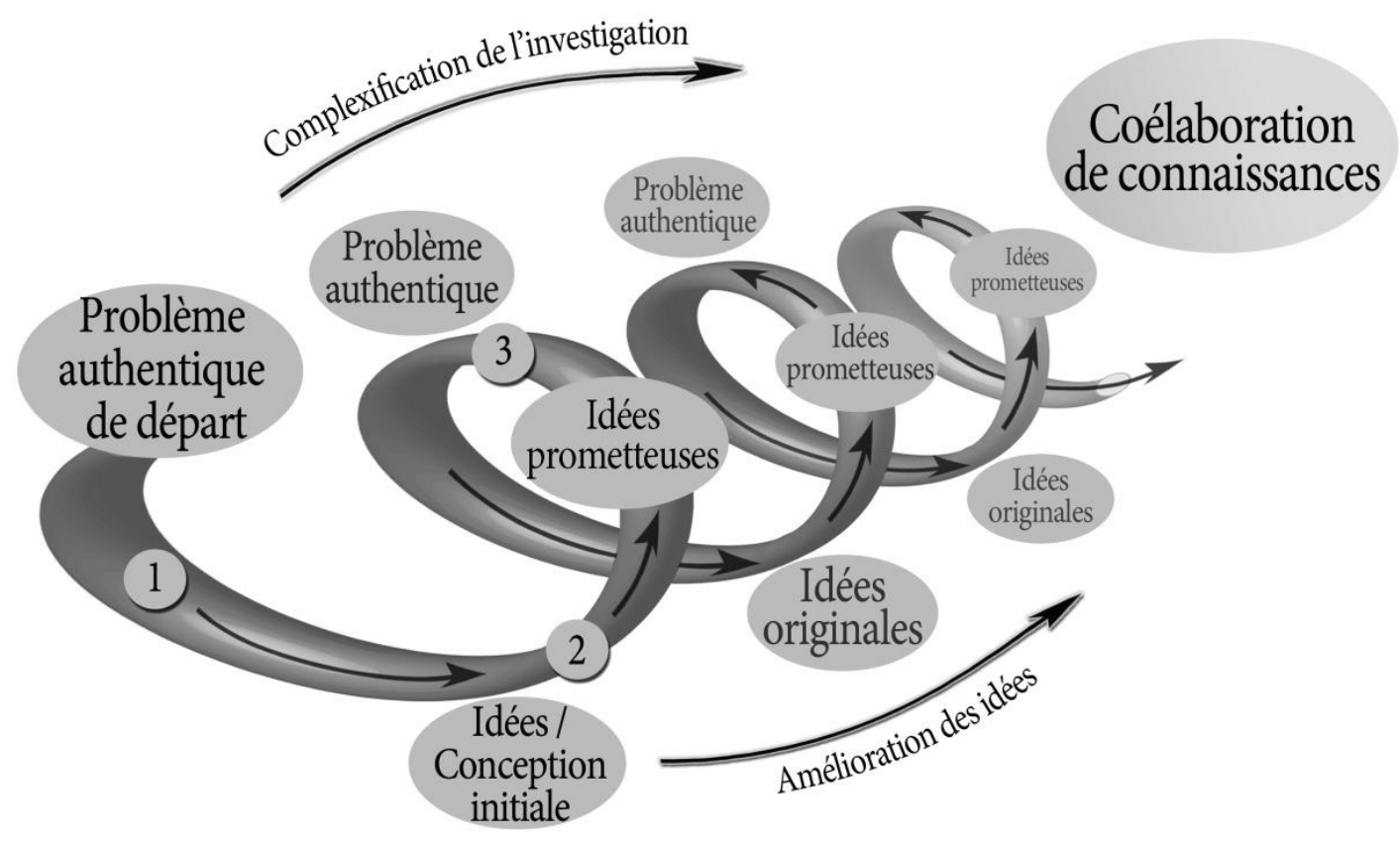

1 - Le problème authentique amène les élèves à formuler des théories, à exprimer leurs idées sur un sujet.

2 - L'utilisation de l'outil IPROM permet aux élèves de sélectionner des idées prometteuses.

3 - Le repérage des idées à approfondir amène de nouvelles questions, plus pointues.

Figure 1 - Le modèle de l'utilisation de l'outil IPROM lors de l'élaboration du discours collectif 
La création de connaissances (Bereiter, 2002; Paavola et al., 2004) suppose que l'élève peut participer à la création de connaissances individuelles et collectives. La création de connaissances renvoie ici au fait que les élèves peuvent conceptualiser, organiser, structurer, créer de la connaissance nouvelle pour leur communauté (Bereiter et Scardamalia, 2010). À cet égard, Bereiter et Scardamalia (2003) distingue la présence de deux modes d'apprentissage en classe. Le belief mode réfère au travail en classe à partir d'éléments "connus" et reconnus. L'élève ne découvre pas, car les faits sont présentés comme étant tels. L'élève n'a pas alors à découvrir le sens du concept qu'il étudie. Le belief mode se rattache davantage à l'enseignement traditionnel où l'on fait peu appel à la créativité et à la capacité d'innovation de l'enfant. Le design mode renvoie, quant à lui, à la théorie de la construction sociale des connaissances. La création/coélaboration de connaissances vise d'abord la construction de connaissances nouvelles par la communauté d'apprenants qui travaille alors sur des problèmes authentiques et qui s'engage dans un processus d'amélioration des idées émises par les participants. Les concepts de coélaboration de connaissances et de design mode mettent également en lumière les concepts d'apprentissage intentionnel (Bereiter et Scardamalia, 1989) dont la mise en application s'exécute par l'élaboration de questions authentiques de la part des apprenants combinée à la construction sociale de connaissances dans un environnement collaboratif. L'essence de ce processus que le design mode supporte est l'amélioration des idées, dans le but de faire avancer la connaissance de la communauté sur une question ou un problème donné (Bereiter et Scardamalia, 2003). C'est le jugement collectif en regard de ce qui est prometteur et le désir de la communauté de pousser plus loin l'ídée en question qui lui donne toute sa portée. Ayant pour pierre angulaire le processus d'amélioration des idées, les idées originales des élèves sélectionnées comme étant prometteuses amènent de nouveaux problèmes authentiques, et un processus d'approfondissement de connaissances s'amorce. Les idées sont l'essence du discours, elles sont constituées des théories, concepts ou explications contenus dans les différentes contributions des élèves au discours collectif. Les idées sont donc la matière brute, le processus de coélaboration puise à celle-ci afin de se raffiner, de se structurer et de s'approfondir.

\subsubsection{Les façons de contribuer au discours collectif}

Les contributions écrites dans le discours collectif prennent différentes formes et viennent le complexifier de différentes façons. Elles sont également étroitement liées à l'intention que lui donne l'élève, ce qu'il choisit d'apporter au discours dans le but 
de le faire avancer. Le processus d'élaboration du discours collectif, la coélaboration de connaissances, est en fait l'amalgame des différentes contributions appelées à former un artéfact conceptuel une fois que le travail sera terminé. Les contributions doivent avoir une certaine valeur pour la communauté et l'élève se le fait rappeler par l'enseignant qui les guide dans le processus de coélaboration de connaissances. De plus, les contributions doivent être complémentaires afin de faire avancer le discours sinon celui-ci va stagner ou s'éteindre (Chuy, Resendes, et Scardamalia, 2010). De plus, dans une communauté d'apprenants en réseau qui se veut une communauté de coélaboration de connaissances, il est de la responsabilité de chacun des apprenants de contribuer aux savoirs collectifs par le partage de ses idées, de ses conceptions initiales ou de son expertise (Scardamalia, 2002). Ainsi la résolution d'un problème et l'avancement du savoir reviennent donc à l'effort de chacun des participants. Cet engagement mutuel des apprenants est au cœur de la complémentarité des contributions et, pour un participant, il importe de considérer les compétences des autres aussi bien que les siennes afin de contribuer de façon efficace au discours (Zhang et Sun, 2008). Étant donné les multiples façons de contribuer au discours collectif, il est parfois difficile de cerner l'ensemble des intentions derrière les contributions des élèves. À ce sujet, Bereiter et Scardamalia (1987) ainsi que Chuy et al. (2010) soutiennent que les élèves engagés dans un processus de coélaboration de connaissances contribuent au dialogue du groupe de façon distincte, et cela passe par l'expression de leurs idées, théories et opinions, par la référence à des faits, par le recours à des analogies et par la synthèse des idées.

\subsection{L'outil d'écriture en collaboration: le Knowledge Forum}

Le Knowledge Forum $(\mathrm{KF})^{2}$ sert de support à l'élaboration du discours écrit et à la négociation de sens qui peut $s^{\prime} y$ produire. Le KF est une plate-forme de type forum électronique qui a été développée dans le but de rendre accessible, en le démocratisant, le processus de création de connaissances (Bereiter et Scardamalia, 2010). Le KF s'appuie sur des principes de coélaboration de connaissances et son design se veut un support à l'élaboration d'un discours collectif en mettant à la disposition des apprenants certaines fonctionnalités pour soutenir l'écriture et la métacognition. L'écriture en collaboration est comprise comme un exercice d'écriture transformatif qui permet aux idées de sans cesse se raffiner par la négociation de sens entre pairs, et sous la guidance de l'enseignant. Ce sont les rétroactions et évaluations formatives entre pairs qui vont surtout permettre à chacun d'occuper une place, une responsabilité dans le processus d'avancement des

Téléaccessible à l'adresse <www.knowledgeforum.com>. 
connaissances collectives. C'est d'ailleurs dans cette optique que les fonctionnalités du KF ont été développées (Scardamalia, 2004).

\subsection{L'outil des idées prometteuses (IPROM): nouvelle fonctionnalité du Knowledge Forum}

L'outil des idées prometteuses (IPROM) est un outil récent disponible sur le Knowledge Forum. Cet outil inclut deux composantes: 1) un surligneur permettant aux apprenants de sélectionner dans le discours collectif les idées qu'ils trouvent prometteuses (voir figure 3), c'est-à-dire les plus porteuses de sens pour la compréhension collective, celles sur lesquelles ils veulent approfondir pour l'avancement de leur investigation collective et 2) une liste hiérarchisée de toutes les idées sélectionnées dans une même perspective en fonction de la fréquence du groupe de mots sélectionné par les élèves (voir figure 2). Cette liste permet de choisir des idées prometteuses afin de les exporter vers une nouvelle perspective. Cela permet à la communauté de voir quelles sont les idées les plus prometteuses, voire à éventuellement approfondir dans leur investigation collective. Comment cet outil, qui a été conçu afin de permettre aux élèves de se centrer sur les idées (Chen et al., 2011), sera-t-il utilisé par les élèves? Étant donné l'état actuel du développement de l'outil ainsi que l'innovation pédagogique en cause, notre étude cherchera à comprendre l'usage qui peut être actuellement fait de cette fonctionnalité dans la pratique d'écriture de classes du primaire. Les figures suivantes présentent l'outil IPROM à son stade actuel de développement dans le KF.

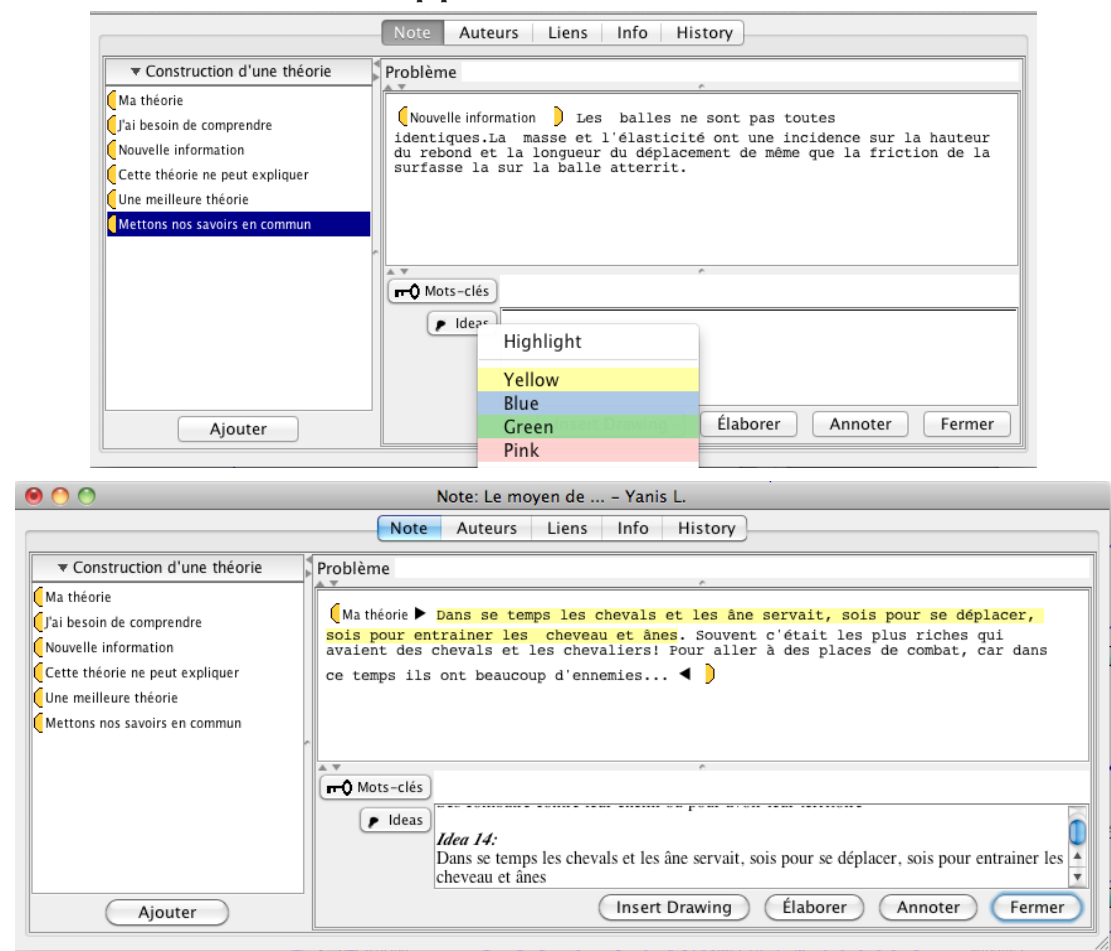

Figure 2 - Une contribution d'élève et le repérage d'idées prometteuses 


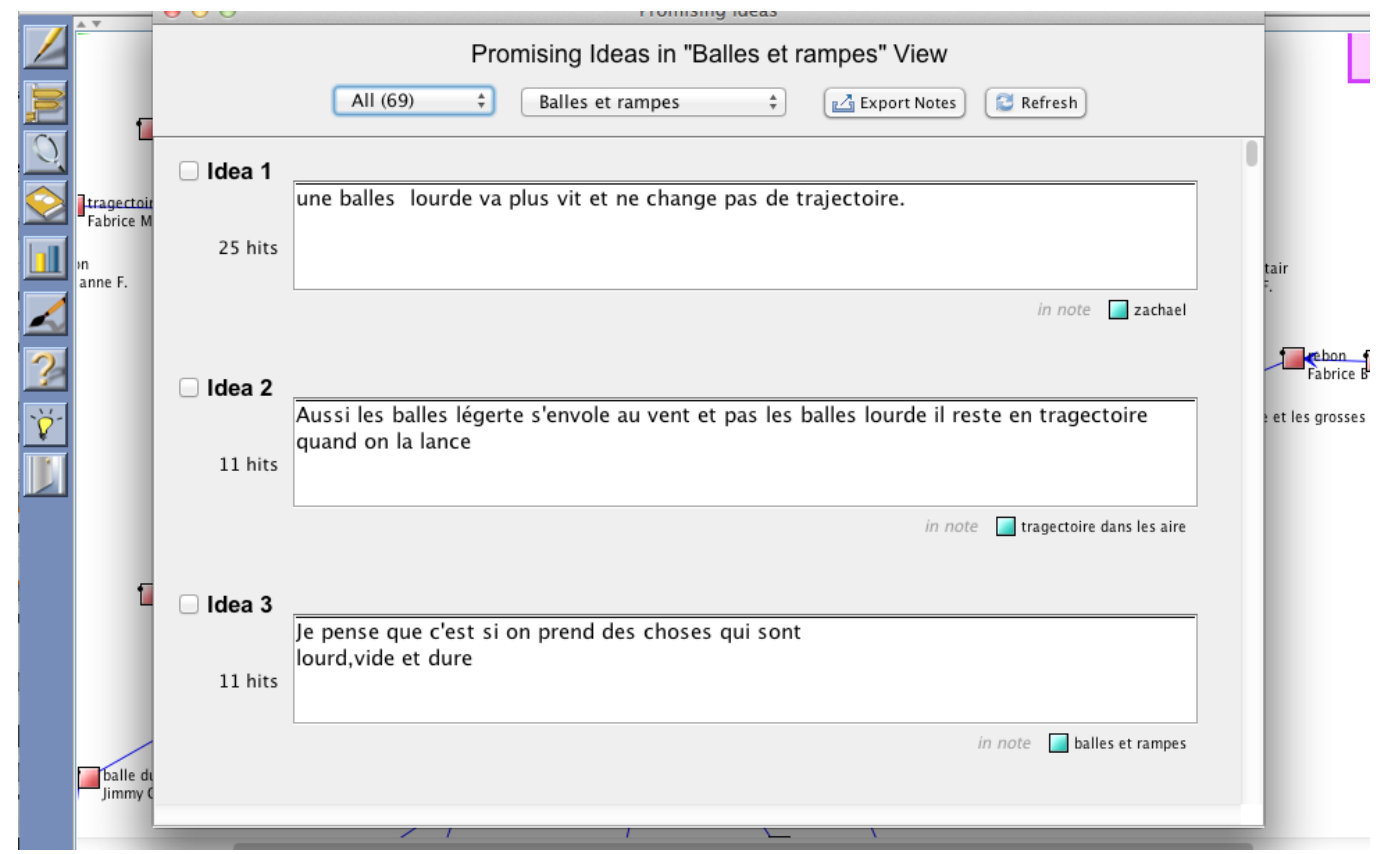

Figure 3 - Les idées prometteuses sélectionnées par les élèves, classées par nombre de fois où elles ont été surlignées

L'outil IPROM vise donc, à l'aide de ses fonctionnalités, à mettre en lumière le rôle primordial que tiennent les idées des élèves dans la coélaboration de connaissances en les plaçant à l'avant-plan (Chen et al., 2011; Chen et al., 2012a; Chen et al., 2012b, Chen et al., 2015). Cette étude est donc un premier pas dans la recherche, du moins en milieu francophone, sur les apports de ce nouvel outil à la coélaboration de connaissances, mais aussi à la compréhension de la façon dont les élèves utilisent leurs idées dans leur processus d'écriture tant collectif qu'individuel. Nous avons tenté de comprendre les perceptions des élèves quant au concept d'idée prometteuse et l'utilisation faite de l'outil. Les conditions se trouvaient réunies pour que les questions de recherche suivantes puissent être formulées:

- Comment les élèves décrivent-ils leur usage de l'outil IPROM pour la sélection des idées prometteuses dans leur discours collectif?

- Quelles façons de contribuer au discours collectif ont été sélectionnées comme étant des idées prometteuses par ceux-ci? 


\section{Méthodologie}

\subsection{Les classes participantes}

Quatre classes du primaire faisant partie de l'initiative de l'École éloignée en réseau ont été ciblées pour participer à cette étude. Elles comptaient deux dyades d'enseignantes (tableau 1), une du $2^{\mathrm{e}}$ cycle et une autre du $3^{\mathrm{e}}$ cycle. En collaboration avec les enseignantes impliquées, nous avons ciblé les élèves de manière à assurer une diversité et ainsi tenir compte, au mieux, des différents profils d'élèves présents dans une classe quant aux 1) résultats scolaires, 2) à la participation en classe (la façon dont les élèves communiquent en classe) et 3) à leur profil de participation sur le KF. Le profil scolaire, rapporté par les enseignantes, permet de nous éclairer sur l'élève et sa motivation, sur son rapport à l'apprentissage et sur les difficultés identifiées par les enseignantes. Il nous importait que les élèves choisis représentent l'éventail des élèves présents dans une classe (Group caracteristic sampling, Patton, 2015), autant sur le plan des résultats scolaires que sur le plan de la participation en classe et sur le KF. À la lumière de ces critères, nous avons rencontré 32 élèves provenant des quatre classes impliquées dans le projet.

\section{Tableau 1}

Classes participantes à l'étude

\begin{tabular}{llll}
\hline Dyades (cas) & Classes & $\begin{array}{l}\text { Niveaux des } \\
\text { élèves }\end{array}$ & $\begin{array}{l}\text { Nombre d'élèves } \\
\text { par classe }\end{array}$ \\
\hline 1 & A & $5^{\mathrm{e}}$ et $6^{\mathrm{e}}$ années & 24 élèves \\
& $\mathrm{B}$ & $6^{\mathrm{e}}$ année & 18 élèves \\
2 & $\mathrm{C}$ & $3^{\mathrm{e}}$ et $4^{\mathrm{e}}$ années & 21 élèves \\
& $\mathrm{D}$ & $3^{\mathrm{e}}$ et $4^{\mathrm{e}}$ années & 17 élèves \\
\hline
\end{tabular}

\subsection{Déroulement}

Le projet s'est déroulé de septembre à novembre 2012 dans les classes A et B et de février à avril dans les classes $C$ et $D$. L'étudiante-chercheure associée au projet a été en classe avec les enseignantes pour présenter l'outil des idées prometteuses (IPROM) et son fonctionnement aux élèves. Les projets vécus en classe ont été d'abord élaborés par les enseignantes pour ensuite être adaptés en fonction de l'utilisation de l'outil IPROM après que les enseignantes aient accepté de participer à l'étude. Les classes ont d'abord vécu une discussion de groupe sur les thèmes qui allaient être abordés, soit le Moyen Âge 
pour les classes A et B et les balles pour les classes C et D. Ensuite, les élèves ont été invités à contribuer sur le KF, à y écrire leurs premières idées. Ils ont ensuite utilisé diverses ressources (recherches sur Internet, manuels de classe, livres, etc.) pour améliorer leurs idées de façon collective. Après quoi, ils ont utilisé l'outil des idées prometteuses (IPROM). S'en sont suivi d'autres recherches des élèves, en lien avec les idées à approfondir et d'autres discussions en classe dans le but de réaliser un texte informatif sur le Moyen Âge (classes $A$ et $B$ ) et de créer une balle (classes $C$ et $D$ ). À la suite de la réalisation de leur projet respectif, les élèves ciblés ont été rencontrés en entretien. Notre équipe a ensuite débuté l'analyse des transcriptions et des idées sélectionnées sur le KF.

\subsection{Instrumentation}

La présente étude de cas (Merriam, 1998; Yin, 2013) vise à documenter la façon dont les élèves décrivent leur usage de l'outil IPROM et les façons de contribuer sélectionnées comme étant des idées prometteuses par les deux dyades, c'est-à-dire des classes A et B et des classes C et D. Notre étude de cas se veut interprétative (Merriam, 1998) au sens où nous cherchons à illustrer et analyser l'utilisation de l'outil IPROM dans des classes du primaire en faisant ressortir les similitudes et différences entre les élèves des deux dyades de classes quant à leur définition d'une idée prometteuse (ce qu'ils en disent) et les façons de contribuer sélectionnées comme étant des idées prometteuses (ce qu'ils ont fait). Nous cherchons également à mettre en relief les possibles écarts ou rapprochements pouvant être faits entre les différents contextes d'utilisation de l'outil IPROM.

Afin de mettre en lumière la compréhension du concept d'idée prometteuse et les façons de contribuer sélectionnées comme étant des idées prometteuses, nous avons eu recours à l'entretien semi-dirigé. L'entretien semi-dirigé est une technique de collecte de données permettant le dialogue et la coconstruction de sens avec les participants de l'étude (Savoie-Zajc, 2003). Nous avons privilégié cette méthode afin de saisir la compréhension que les élèves avaient des idées prometteuses. L'usage de l'outil IPROM et la conception d'une idée prometteuse des élèves sont au cœur des analyses du discours recueilli lors des entretiens semi-dirigés.

\subsection{L'analyse de données}

Pour chacun des élèves rencontrés lors des entretiens, nous avons analysé les verbatims portant sur le concept d'idée prometteuse. Nous avons analysé les transcriptions 
d'entretien en relevant de façon thématique les classifications des descriptions de l'usage de l'outil IPROM pour la sélection des idées prometteuses (Chen et al., 2012a) (tableau 2).

Tableau 2

La classification des usages décrits par les élèves de l'outil IPROM

\begin{tabular}{ll}
\hline Description & Exemples \\
\hline Un mot important, un mot clé & $\begin{array}{l}\text { Ca sert à garder le mot qui est le plus } \\
\text { important. C'est surtout le mot le plus } \\
\text { important dans la question ou la réponse, ça } \\
\text { nous aide à comprendre qu'est-ce qu'il veut } \\
\text { dire. }\end{array}$ \\
$\begin{array}{l}\text { Une idée que l'on pense vraie, } \\
\text { Je regarde et je sais celles qui sont bonnes et } \\
\text { importante }\end{array}$ & $\begin{array}{l}\text { correctes. Je me pose des questions, je les } \\
\text { utilise et je les souligne. }\end{array}$ \\
$\begin{array}{l}\text { Une idée intéressante, une } \\
\text { nouvelle idée }\end{array}$ & $\begin{array}{l}\text { C'est une idée qui est assez bonne et qui a de } \\
\text { la logie, et importante. } \\
\text { Une idée qui aide, qui permet } \\
\text { d'aller plus loin. Quelque prometteuse c'est une idée qui } \\
\text { chose à comprendre qui } \\
\text { permet une action future. }\end{array}$ \\
\hline
\end{tabular}

Le tableau 2 nous a permis d'attribuer un usage de l'outil IPROM à chacun des élèves rencontrés (32). Nous avons utilisé un logiciel d'analyse qualitative (TAMS Analyzer) afin de repérer et coder les unités de sens thématiques en lien avec les différentes conceptions des idées prometteuses des élèves. Dans une approche d'analyse de contenu (Krippendorff, 2012), nous avons isolé les idées prometteuses sélectionnées par les élèves à l'aide de l'outil IPROM. Les idées prometteuses ont fait l'objet d'une analyse qualitative en ce qui a trait à leur nature, à la façon de contribuer des élèves (Chen et al., 2012b; Chuy et al., 2010) à l'aide du logiciel d'analyse qualitative TAMS Analyzer. Pour ce faire, nous nous sommes principalement basées sur la grille utilisée par Chen et al. (2012b) contenant les trois principales catégories (Faits, Théories, Questions) auxquelles nous avons ajouté des sous-catégories permettant d'aller plus en profondeur quant à la façon de contribuer au discours. Nous avons aussi ajouté les différents types de questions afin d'en clarifier la nature (questions requérant une réponse à développement court, questions requérant une réponse à développement long et questions orientées sur le processus) proposés par Hmelo-Silver et Barrows (2008). 
Tableau 3

Les différentes façons de contribuer au discours inspiré de

Chuy et al. (2010), Chen et al. $(2011 ; 2012 b)$

\begin{tabular}{ll}
\hline Types d'idées & Façons de contribuer au discours \\
\hline Faits & Apporte un nouveau fait \\
& Répète un fait \\
& Remet en question un fait \\
& Soutient un fait \\
\hline Théories & Apporte une nouvelle théorie \\
& Soutient une théorie \\
& Remet en question une théorie \\
& Synthétise des théories présentes dans le discours \\
\hline Questions & Questions requérant une réponse à développement court \\
& Questions requérant une réponse à développement long \\
& Questions orientées sur le processus \\
\hline
\end{tabular}

Le tableau 3 comprend trois catégories de conception d'une idée prometteuse par les élèves, soit 1) "une idée vraie", 2) "une idée qui est probablement vraie, qui fonctionne", 3) "une question prometteuse". Les élèves ont sélectionné 118 idées prometteuses différentes. Les idées prometteuses ont été considérées comme des unités de sens, puisqu'elles se distinguent sur le plan physique (Krippendorff, 2012) de par leur sélection. Afin de mesurer la compréhension mutuelle des catégories pour une même unité de sens, nous avons utilisé le coefficient d'accord interjuge alpha de Krippendorff (2012) sur $16 \%$ des idées prometteuses sélectionnées (118) et nous avons obtenu $\alpha=0,8455$. Les analystes ont un niveau d'accord plus élevé que la norme minimale qui est de 0,80.

\section{Résultats}

\subsection{Les usages décrits de l'outil IPROM par les élèves}

\subsubsection{D’abord, un usage pour s'aider soi-même}

Les élèves des classes $A$ et $B$ ont souligné que le fait d'avoir accès aux idées que les autres ont surlignées les a aidés à mieux comprendre. L'Élève B-2 le souligne en ces termes: Ça aidait à garder les idées, les informations, on peut aller voir les réponses des autres, les choses que les autres ont surlignées, ça nous aidaità comprendre les choses. L'Élève A-5 ajoute: 
C'était plus facile de surligner, ça m'aidait plus à comprendre parce que les autres allaient écrire, je surlignais les choses que je ne comprenais pas, ils allaient me les expliquer, alors j'allais plus comprendre. Des élèves des classes $\mathrm{C}$ et $\mathrm{D}$ rapportent avoir surligné dans les contributions de leurs pairs et d'avoir pu facilement consulter les idées choisies par les autres élèves. L'Élève C-2 exprime: Tu peux voir les idées prometteuses, si tu ne t'en souviens plus, c'est comme une mémoire, tu peux aller voir. Certains élèves relèvent que l'outil IPROM les aide dans leurs apprentissages. Par exemple, I'Élève C-7 mentionne: J'apprends des nouvelles choses au fur et à mesure. Ça m'aide beaucoup.

\subsubsection{Un usage timide au service du discours collectif}

Les élèves ont sélectionné des idées prometteuses à l'intérieur des contributions de leurs pairs et ont consulté les idées sélectionnées par leur classe, certains s'en sont ensuite inspirés afin de contribuer au discours collectif. Ils les ont utilisées pour orienter leurs recherches, et pour poursuivre leur(s) investigation(s). Pour les classes A et B, I'Élève B-6 soutient que: On peut l'utiliser pour voir ce que les gens aiment le plus, pour savoir sur quoi on va travailler, continuer les projets par rapport à ce qui a été surligné. L'Élève A-1 ajoute: C'est sûr que c'est différent, les enseignantes peuvent savoir ce qui nous intéresse. Ce propos d'élève laisse sous-entendre ici le rôle de l'enseignante dans l'orientation et l'organisation du travail collectif futur et en fonction des idées prometteuses. Dans les classes C et D, l'outil a servi à l'élaboration du discours collectif en permettant à certains élèves de consulter les idées prometteuses sélectionnées pour s'assurer que leur contribution soit pertinente, comme le précise l'Élève C-5: Oui je me suis servi de ça, c'est sûr que ça aidait, ça nous aidait à vraiment choisir ce qu'on allait dire, pour ne pas se répéter.

4.1.3 Un usage basé sur une compréhension de l'idée prometteuse comme étant au service de chacun ou du collectif

Plusieurs élèves des différentes dyades décrivaient les idées prometteuses comme étant des mots importants ou des mots clés dans les contributions de leurs pairs, l'Élève C-6 explique: C'est surtout le mot le plus important dans la question ou la réponse. D'autres les percevaient comme étant des idées ou des informations importantes:

Élève D-9: Je lisais et ce que je trouvais, qui était le meilleur, je le surlignais.

Élève B-7: Souligner, je dirais si vous trouvez des affaires intéressantes, tu peux aller les souligner, et choisir la couleur jaune et trouver ce qui est important dans ce que les gens ont expliqué. 
Élève A-3: Je vais lire au complet le texte, et je vais voir c'est quoi le contexte, les informations importantes, lorsque c'est bon de le savoir.

Quelques élèves percevaient davantage les idées prometteuses comme étant au service du collectif et les voyaient comme des idées intéressantes ou nouvelles. Ainsi l'explique I'Élève A-2: J'ai regardé et j'ai trouvé que c'était intelligent avec des questions ou ce sont des idées que je connais pas, que je voudrais plus en savoir. L'Élève B-6 ajoute: ...je surligne ce qui serait intéressant pour la classe. Aussi, un élève sur cinq définissait les idées prometteuses comme étant au service de la poursuite de l'investigation collective. Par exemple, pour l'Élève C-6, les idées prometteuses, c'est ce qui nous promet quelque chose, promettre quelque chose ça veut dire que ça peut nous aider à faire quelque chose. Dans les deux cas, les élèves ont surtout perçu les idées prometteuses comme une aide individuelle qui leur permettait de mettre en lumière les mots clés ou les informations importantes. De plus, l'outil IPROM leur permet de consulter facilement les idées de leurs pairs et de cibler les idées importantes dans le discours collectif. Un usage timide de l'outil a été fait au service du discours collectif, certains élèves percevaient davantage les idées prometteuses comme des idées intéressantes qui pouvaient les amener plus loin, ce qui pouvait leur permettre de faire, d'orienter et de faire avancer le discours collectif. Ces compréhensions différenciées des élèves ne sont pas sans influencer la sélection des idées qu'ils identifient comme étant prometteuses.

\subsection{Façons de contribuer au discours sélectionnées par les élèves}

Les classes A et B ont travaillé à en apprendre davantage sur la période historique du Moyen Âge afin d'élaborer un texte informatif et une maquette représentant un pan de cette époque. Les classes des enseignantes $C$ et $D$ ont quant à elles travaillé à l'élaboration d'une balle en touchant aux concepts de trajectoire, rebond et vitesse. Ces quatre classes ont utilisé le KF afin que les élèves élaborent leurs idées de façon collective. Ainsi, en nous référant aux grilles de Chuy et al. (2010) et Chen et al. (2011, 2012b), 118 idées prometteuses différentes ont été sélectionnées dans les classes des enseignantes A et B. Plusieurs de ces 118 idées distinctes ont été sélectionnées de nombreuses fois par différents élèves (94, soit $80 \%$ des idées sélectionnées), ce qui donne un total de 453 idées sélectionnées. Pour les classes des enseignantes $C$ et $D$, on compte 70 idées prometteuses différentes qui ont été sélectionnées, dont $91 \%$ ont été sélectionnées plus d'une fois par les élèves dans leur discours collectif, ce qui mène à un total de 274 idées prometteuses. 


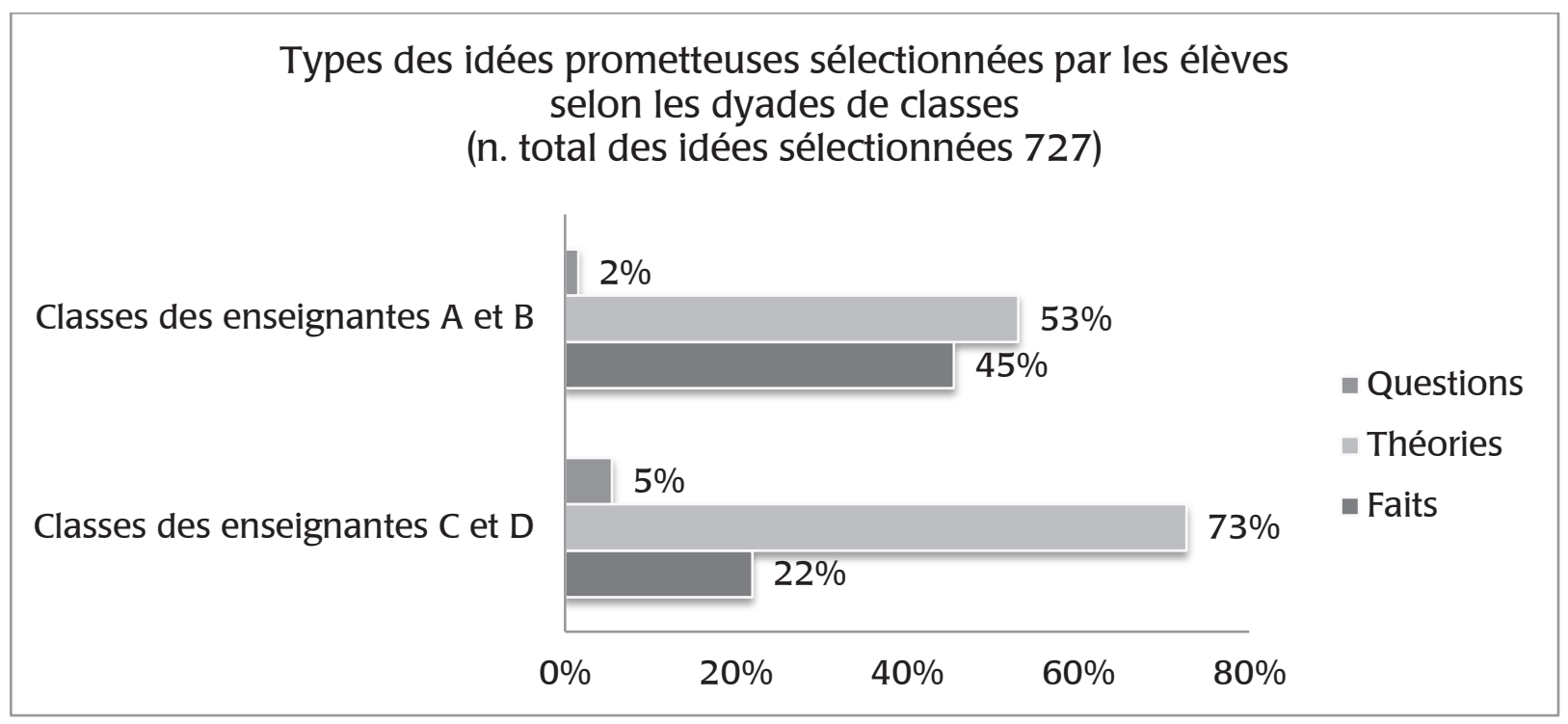

Figure 4 - Les types d'idées prometteuses sélectionnées par les élèves

\section{Classes A et B (Activité d'apprentissage: le Moyen Âge)}

Ainsi, une idée sélectionnée sur deux (53\%) se veut une "Théorie" dans les façons de contribuer au discours. On constate également que des "Faits" ont également été sélectionnés comme étant des idées prometteuses et représentent $45 \%$ des idées prometteuses. On remarque que peu de questions ont été sélectionnées, car elles ne représentent que $2 \%$ des idées prometteuses.

\section{Classes C et D (Activité d'apprentissage: les balles et les rampes)}

Ici, les façons de contribuer sélectionnées comme étant des idées prometteuses sont majoritairement de type "Théories" et représentent trois idées sur quatre (73\%). Les "Faits" sont présents dans une proportion d'une idée sélectionnée sur cinq ( $22 \%)$. On constate ici que les questions représentent encore une fois peu d'idées sélectionnées (5\%).

C'est dire que les élèves des classes A et B (53\%) et des classes C et D (73\%) ont sélectionné majoritairement des idées prometteuses de type "Théories", mais dans des proportions différentes. Ceci révèle que les élèves des classes des enseignantes $C$ et $D$ ont sélectionné davantage d'idées prometteuses de type "Théories" émanant de leurs pairs que des affirmations ou des faits provenant de différentes sources d'autorité. Les "Faits", quant à eux, sont beaucoup plus présents dans les idées sélectionnées par les élèves des classes des enseignantes A et B (45\%) que dans celles des classes des enseignantes $C$ et $D(22 \%)$. Ce phénomène pourrait s'expliquer par le fait que les "Faits" sont aussi plus présents dans les contributions des élèves des classes A et B. Ainsi l'activité 
sur le Moyen Âge, traitant d'un sujet historique, aurait pu amener les élèves à considérer davantage les faits historiques que les explications et les théories, afin de répondre à la question de départ et la production attendue, soit un texte informatif. De plus, les élèves des classes $C$ et $D$ ont considéré davantage de questions ( $5 \%$ ) comme étant des idées prometteuses pouvant les amener plus loin que les classes des enseignantes A et B (2\%). Ceci pourrait s'expliquer par un nombre plus grand de questions posées par les élèves dans l'ensemble du discours collectif des classes $C$ et $D$ qui travaillaient sur l'élaboration d'une balle. Les élèves auraient été plus enclins à poser des questions sur les façons de faire de leurs pairs pour négocier la création de leur propre balle (matériaux utilisés, formes, poids).

Toujours selon une grille inspirée de Chuy et al. (2010) et Chen et al. (2011; 2012b), la figure 5 présente les différentes façons de contribuer au discours qui sont les plus souvent perçues comme des idées prometteuses par les élèves.

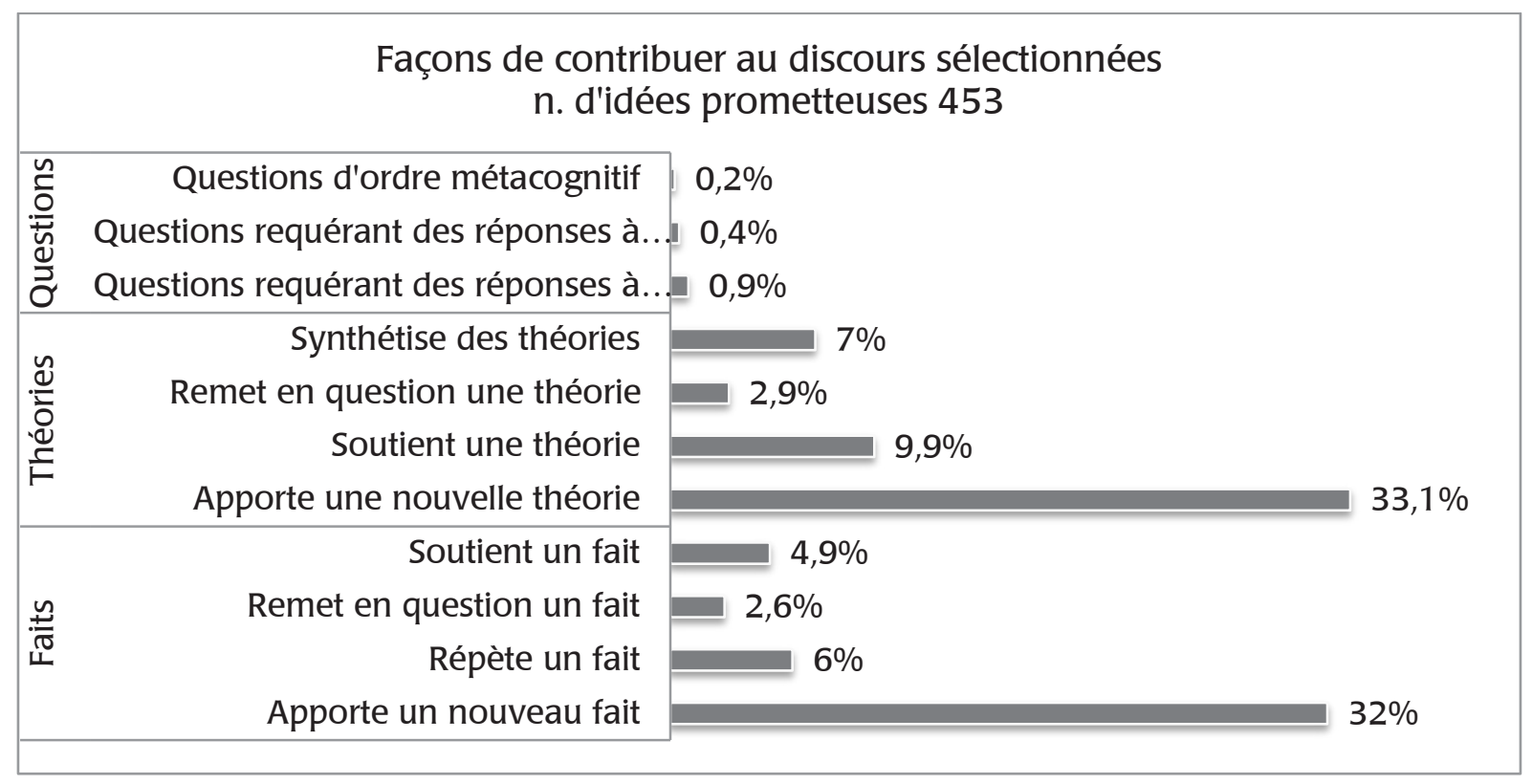

Figure 5 - Les façons de contribuer au discours sélectionnées comme étant des idées prometteuses pour les classes des enseignantes A et B

Pour les classes des enseignantes A et B, les idées prometteuses de type "Théories" sélectionnées par les élèves se répartissent entre les façons de contribuer au discours suivantes: "Apporte une nouvelle théorie» $(33,1 \%)$, "Synthétise des théories” (7\%), "Soutient une théorie" $(4,9 \%)$, et "Remet en question une théorie" (2,9\%). Du côté des "Faits", on remarque que c'est "Apporte un nouveau fait" qui est le plus sélectionné avec $32 \%$ des idées. Toujours concernant les "Faits", "Répète un fait" représente 9,9\% des idées, "Soutient un fait" représente $4,9 \%$ des idées et "Remet en question un fait" 
représente $2,9 \%$ des idées. On remarque que «Apporte une nouvelle théorie» et «Apporte un nouveau fait» représentent tous les deux une idée sur trois (33,1\% et $32 \%$ ). Bien que les questions ne représentent que $2 \%$ des idées prometteuses, on remarque qu'elles se partagent entre les différents types de questions, "Questions d'ordre métacognitif» $(0,2 \%)$, "Questions requérant une réponse à développement long» $(0,4 \%)$ et que ce sont les "Questions requérant une réponse à développement court» $(0,9 \%)$ qui sont le plus souvent sélectionnées par les élèves.

\section{Tableau 4}

Exemples des façons de contribuer au discours sélectionnées comme étant des idées prometteuses par les élèves des classes A et B

\begin{tabular}{|c|c|c|}
\hline $\begin{array}{c}\text { Types } \\
\text { d'idées }\end{array}$ & $\begin{array}{l}\text { Façon de contribuer au } \\
\text { discours }\end{array}$ & $\begin{array}{c}\text { Moyen Âge } \\
\text { (classes A et B, } 3^{\mathrm{e}} \text { cycle du primaire) }\end{array}$ \\
\hline \multirow{4}{*}{$\begin{array}{c}\text { Faits } \\
(45,5 \%)\end{array}$} & Apporte un nouveau fait & $\begin{array}{l}\text { - Les rois vivaient dans des châteaux en } \\
\text { bois ou en pierres. } \\
\text { - Ça se passait } 500 \text { ans après Jésus-Christ. }\end{array}$ \\
\hline & Répète un fait & $\begin{array}{l}\text { Les personnes importantes vivaient dans des } \\
\text { châteaux en bois ou en pierres. }\end{array}$ \\
\hline & Remet en question un fait & $\begin{array}{l}\text { D'après moi ça se situait environ } 800 \text { ans } \\
\text { après Jésus-Christ. }\end{array}$ \\
\hline & Soutient un fait & $\begin{array}{l}\text { Il y avait des petits villages dotés de plein de } \\
\text { maisons, de fermes, et généralement d'un } \\
\text { château... Les châteaux appartenaient aux } \\
\text { nobles comme les rois et reines, aux ducs, } \\
\text { etc.? }\end{array}$ \\
\hline \multirow[b]{3}{*}{$\begin{array}{l}\text { Théories } \\
\text { (53\%) }\end{array}$} & Apporte une nouvelle théorie & $\begin{array}{l}\text { Il existait, je pense, des télévisions à } \\
\text { manivelle. Des lanternes à l'huile. Les } \\
\text { châteaux étaient faits en terre et en boue et } \\
\text { de roches. II y avait des poêles en terre, de } \\
\text { paille, de roches et d'argile. }\end{array}$ \\
\hline & Soutient une théorie & $\begin{array}{l}\text { Je pense qu'il y avait des châteaux et les } \\
\text { châteaux avaient des forteresses faites en } \\
\text { bois parce qu'il n'y avait pas de ciment. }\end{array}$ \\
\hline & Remet en question une théorie & $\begin{array}{l}\text { C'est que les châteaux étaient faits en bois, } \\
\text { mais à cause de la guerre ils ont décidé de les } \\
\text { faire en pierres pour que les châteaux de } \\
\text { combat ne se fassent plus détruire. } \\
\text { II avait des cercles (ce sont des croyants). } \\
\text { Dans ce temps, leurs premiers châteaux } \\
\text { étaient faits en bois et à l'évolution les } \\
\text { châteaux se sont construits avec du ciment, } \\
\text { de la brique, de la glaise pour mieux tenir. } \\
\text { Ensuite, ils ont eu des années de paix et ils } \\
\text { ont eu la guerre. }\end{array}$ \\
\hline \multirow{3}{*}{$\begin{array}{l}\text { Questions } \\
(1,5 \%)\end{array}$} & $\begin{array}{l}\text { Questions requérant des } \\
\text { réponses à développement } \\
\text { court }\end{array}$ & Les enfants portaient des armures en bois? \\
\hline & $\begin{array}{l}\text { Questions requérant des } \\
\text { réponses à développement } \\
\text { long }\end{array}$ & $\begin{array}{l}\text { J'aimerais que tu m'expliques, comment } \\
\text { étaient les villages? }\end{array}$ \\
\hline & Questions d'ordre métacognitif & [Pourquoi dis-tu] dans les airs? \\
\hline
\end{tabular}




\section{Classes des enseignantes $C$ et $D$ travaillant sur le projet des balles et des rampes}

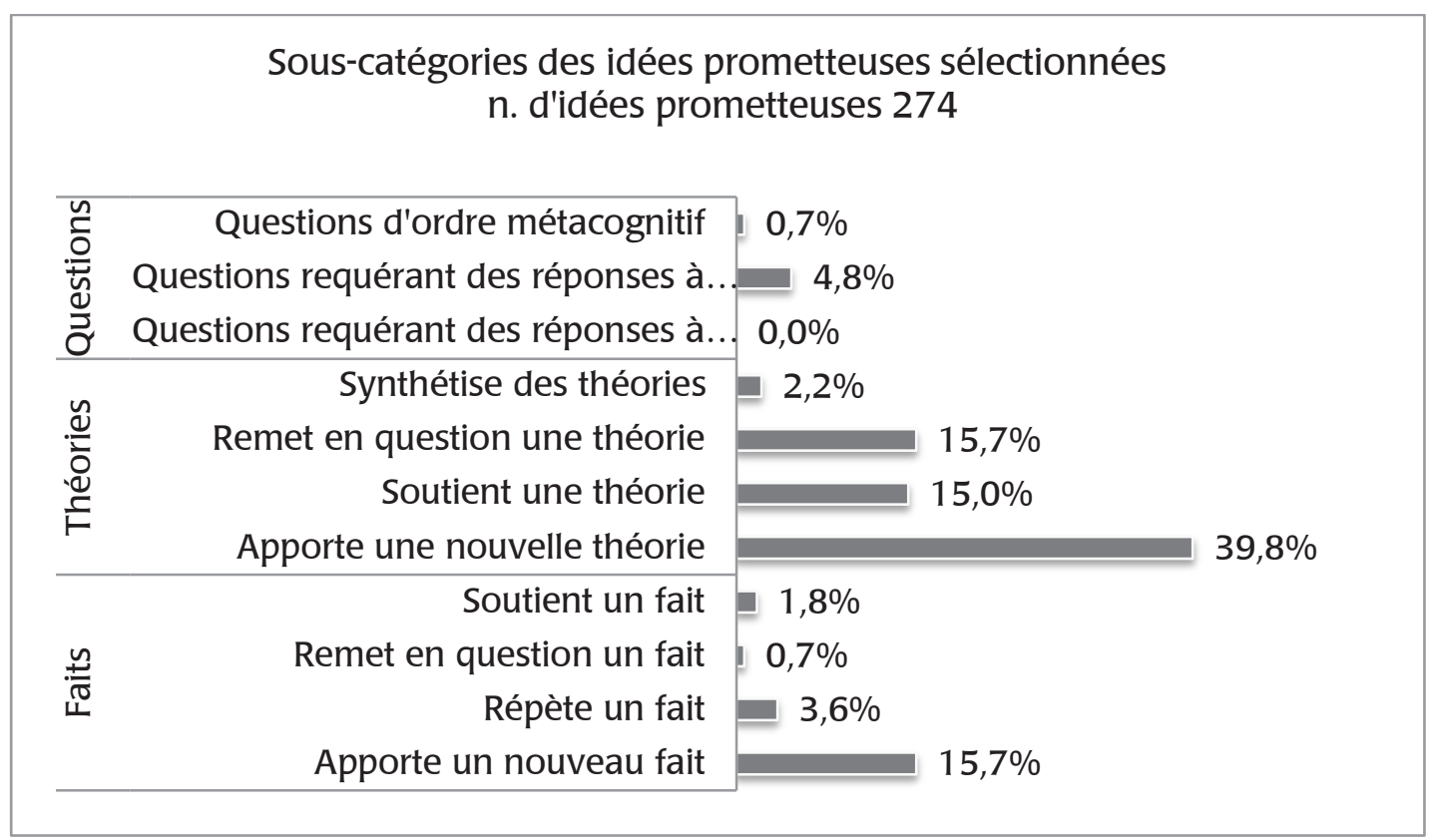

Figure 6 - Les façons de contribuer au discours sélectionnées comme étant des idées prometteuses pour les classes des enseignantes $C$ et $D$

Pour les classes des enseignantes C et D, les façons de contribuer de type "Théories" se répartissent entre "Apporte une nouvelle théorie" (40\%), "Remet en question une théorie» (16\%), "Soutient une théorie» (15\%) et "Synthétise des théories» (2\%). On observe que pour le type "Faits", "Apporte un nouveau fait" représente 15,7\% des idées sélectionnées, "Répète un fait» 3,6 \%, "Soutient un fait» 1,8 \% et "Remet en question les faits" 0,7 \%. Bien que "Apporte une théorie» et "Apporte un nouveau fait» demeurent les façons de contribuer les plus sélectionnées comme étant des idées prometteuses, leurs proportions sont cependant différentes. On constate que la proportion des idées prometteuses qui sont des questions est plus élevée (5\%) et ce sont les questions à développement long qui ont été les plus sélectionnées (4,7 \%).

On constate que, dans les classes des enseignantes $A$ et $B$, «Apporte une nouvelle théorie» (33,1\%) et "Apporte un nouveau fait» (32\%) sont presque nez à nez et sont les façons de contribuer les plus sélectionnées par les élèves comme étant des idées prometteuses. Pour les classes des enseignantes $C$ et $D$, les élèves ont sélectionné davantage des idées qui "Apporte une nouvelle théorie» $(39,8 \%)$ que celles qui «Apporte un nouveau fait» $(15,7 \%)$. On remarque également que les élèves ont sélectionné différentes façons de contribuer en ce qui a trait aux questions. Ainsi, les élèves des classes des enseignantes $A$ et $B$ ont sélectionné quelques questions (2\%) des différents types, mais surtout des questions à développement court $(0,9 \%)$. Les élèves des classes des 
enseignantes $\mathrm{C}$ et $\mathrm{D}$ ont quant à eux, sélectionné surtout des questions à développement long $(4,7 \%)$ comme étant des idées prometteuses.

\section{Tableau 5}

Exemples des façons de contribuer au discours sélectionnées comme étant des idées prometteuses par les élèves des classes C et D

\begin{tabular}{|c|c|c|}
\hline Types d'idées & Façon de contribuer au discours & $\begin{array}{c}\text { Balles et rampes } \\
\text { (classes } C \text { et } \mathrm{D}, 2^{\mathrm{e}} \text { cycle du primaire) }\end{array}$ \\
\hline \multirow{4}{*}{$\begin{array}{c}\text { Faits } \\
(21,8 \%)\end{array}$} & Apporte un nouveau fait & $\begin{array}{l}\text { Le caoutchouc est un bon matériel pour les } \\
\text { rebonds. }\end{array}$ \\
\hline & Répète un fait & $\begin{array}{l}\text { Peut-être en caoutchouc comme la balle en } \\
\text { caoutchouc. }\end{array}$ \\
\hline & Remet en question un fait & $\begin{array}{l}\text { La meilleure balle doit être faite en } \\
\text { plastique. }\end{array}$ \\
\hline & Soutient un fait & $\begin{array}{l}\text { Oui, les balles de tennis rebondissent et les } \\
\text { balles rebondissantes rebondissent. }\end{array}$ \\
\hline \multirow{4}{*}{$\begin{array}{l}\text { Théories } \\
(72,7 \%)\end{array}$} & Apporte une nouvelle théorie & $\begin{array}{l}\text { Une balle lourde va plus vite et ne change } \\
\text { pas de trajectoire. }\end{array}$ \\
\hline & Soutient une théorie & $\begin{array}{l}\text { Aussi les balles légères s'envolent au vent } \\
\text { et pas les balles lourdes, elles restent en } \\
\text { trajectoire quand on la lance. }\end{array}$ \\
\hline & Remet en question une théorie & $\begin{array}{l}\text { Moi, je crois que c'est les balles légères qui } \\
\text { roulent plus vite. }\end{array}$ \\
\hline & Synthétise des théories & $\begin{array}{l}\text { D'après moi la balle qui va rebondir le plus } \\
\text { est une petite balle rebondissante parce } \\
\text { qu'elle est légère. }\end{array}$ \\
\hline \multirow{3}{*}{$\begin{array}{l}\text { Questions } \\
(5,4 \%)\end{array}$} & $\begin{array}{l}\text { Questions requérant des } \\
\text { réponses à développement court }\end{array}$ & Aucun exemple \\
\hline & $\begin{array}{l}\text { Questions requérant des } \\
\text { réponses à développement long }\end{array}$ & À quoi la balle va-t-elle servir? \\
\hline & Questions d'ordre métacognitif & On ne sait pas, peux-tu dire pourquoi? \\
\hline
\end{tabular}

\section{Discussion}

Il ressort de nos données que les élèves ont d'abord décrit leur usage de l'outil IPROM comme une activité individuelle, au sens où les élèves précisent qu'ils ont choisi des idées d'abord pour leur propre compréhension plutôt que pour l'avancement du discours collectif. Ainsi, plusieurs élèves définissaient les idées prometteuses comme des mots 
clés ou des informations importantes, lesquelles leur apportaient de l'aide individuelle. Certains élèves décrivaient aussi les idées prometteuses comme des idées au service du collectif afin d'orienter leur travail et d'éviter la répétition d'idées dans le discours. Ils décrivaient aussi les idées prometteuses comme étant au service du collectif et les voyaient comme des idées intéressantes et des idées leur permettant d'aller plus loin. C'est dire que les élèves avaient des compréhensions différenciées du concept d'idée prometteuse qui n'allaient pas nécessairement de pair avec les usages qu'ils ont faits de l'outil.

De fait, plusieurs élèves ont décrit les idées prometteuses comme des informations importantes, ce qui explique probablement que la majorité des idées sélectionnées était de type "Théories". De plus, on remarque que la plupart des élèves voient l'outil IPROM comme un outil de lecture et d'écriture individuel, dans le but de faire avancer leur propre compréhension, ce qui a pu influencer l'avancement du discours collectif, d'abord centré sur leurs tâches individuelles. En effet, dans les deux activités vécues avec I'utilisation d'IPROM, les élèves ont élaboré un discours collectif, par contre celui-ci était orienté sur une tâche finale à accomplir de façon individuelle, soit la rédaction d'un texte informatif sur le Moyen Âge et la construction de la "meilleure" balle. Les tâches à accomplir en fin d'activité étant de nature individuelle, cela a pu contribuer à ce que les élèves aient utilisé IPROM dans le but de s'aider à accomplir la tâche plutôt qu'à faire avancer leurs connaissances collectives. Les travaux de recherche en apprentissage coopératif ou collaboratif (Brown, 1994; Brown et Campione 1994 ; Brown et al., 1991; Scardamalia, 2002; Scardamalia et Bereiter, 2003) mettent en évidence l'importance d'un produit collectif de manière à établir une cohérence entre le processus et le produit.

Néanmoins, les élèves ont surtout sélectionné des "Théories» (53 \% et 72,7 \%) étant des idées prometteuses dans les deux projets vécus. Ce qui pourrait s'avérer un pas dans le design mode qui renvoie à la théorie de la construction sociale des connaissances (Scardamalia et Bereiter, 2003). La création/coélaboration de connaissances (Paavola et al., 2004) vise d'abord la construction de connaissances nouvelles par la communauté d'apprenants qui travaille alors sur des problèmes authentiques et qui s'engage dans un processus d'amélioration des idées (Bereiter et Scardamalia, 2010; Bereiter, 2002). Une proportion considérable des idées sélectionnées était de l'ordre des "Faits" $(45,5 \%$ et $21,8 \%)$. Les questions, quant à elles, représentent peu d'ídées prometteuses sélectionnées par les élèves $(1,5 \%$ et $5,4 \%)$, ce qui pourrait nous indiquer que les questions de départ ou encore les questions posées durant le discours collectif ne sont pas en soi perçues par les élèves comme étant des idées intéressantes qui 
pourraient les amener plus loin. On observe sensiblement les mêmes proportions dans les façons de contribuer au discours sélectionnées comme étant des idées prometteuses dans l'étude de Chen et al. (2012b), qui portait sur l'utilisation de l'outil IPROM chez des élèves du $2^{\mathrm{e}}$ cycle et du $3^{\mathrm{e}}$ cycle du primaire en milieu anglophone favorisé ${ }^{3}$ et dans laquelle les élèves ont également considéré davantage les "Théories" (63,9\%) comme étant des idées prometteuses. Les auteurs ont également observé qu'une proportion non négligeable des idées prometteuses était des "Faits" (22, $9 \%$ ). Ainsi, I'utilisation d'IPROM, lors de notre étude, en cohérence avec les résultats de l'étude de Chen et al. (2012b), serait davantage orientée vers l'amélioration des idées sélectionnées (Théories) dans le but d'aller collectivement plus loin (design mode), plutôt que vers la sélection des idées importantes (Faits) pour la tâche individuelle à accomplir (belief mode), et ce, bien qu'une pédagogie de la coélaboration de connaissances puisse s'avérer difficile à adopter en classe.

\section{Conclusion}

Cette étude est un premier pas dans l'analyse des usages faits de l'outil IPROM dans un contexte de classes francophones. Elle met en lumière certaines implications pédagogiques pour que l'outil soit mis au service de l'élaboration du discours collectif et du processus de coélaboration de connaissances. Ainsi, bien que l'outil incite les élèves à sélectionner des idées prometteuses, les élèves sélectionnent les théories de leur pairs (design mode) L'utilisation d'IPROM en classe permettrait aux élèves de se centrer sur le processus d'amélioration des idées et d'avancement du discours collectif.

Cette étude a cependant quelques limites. Ainsi, nous n'avons pas documenté le processus individuel des apprenants lors de la sélection des idées ainsi que le soutien offert de la part des enseignantes lors de l'utilisation de l'outil. Les entretiens avec les élèves ont eu lieu après la réalisation du projet, ce qui ne nous offre que peu de détails sur le processus de sélection lui-même. Pour ce faire, il aurait fallu suivre plusieurs élèves lors du travail en classe et leur poser des questions quant aux critères de sélection qu'ils se sont donnés pour la sélection des idées.

Les limites du présent article nous éclairent sur des pistes de recherches futures, à savoir le processus individuel de sélection des idées prometteuses de la part des élèves.

Les classes impliquées dans la présente étude ne se situent pas dans des milieux socioéconomiques très favorisés. En effet, d'après le ministère de l'Éducation, du Loisir et du Sport (2012), l'école des classes A et C a un indice de défavorisation de 10 . Les écoles des classes $B$ et $D$ ont des indices de défavorisation moins élevés avec des taux respectif de 8 et 9 . Les écoles sont classées sur une échelle allant de 1 à 10, le rang 1 étant considéré comme le moins défavorisé et le rang 10 comme le plus défavorisé. 
De plus, nous voulons nous pencher sur le soutien pouvant être offert par les enseignants et autres formateurs afin de guider les apprenants dans le processus de coélaboration de connaissance ayant comme support un forum électronique (Knowledge Forum) par l'entremise de l'outil des idées prometteuses.

\section{Références}

Bereiter, C. (2002). Education and Mind in the Knowledge Age. Mahwah, NJ: Lawrence Erlbaum.

Bereiter, C. et Scardamalia, M. (1987). The Psychology of Written Composition. Hillsdale, NJ: Erlbaum.

Bereiter, C. et Scardamalia, M. (1989). Intentional learning as a goal of instruction. In L.B. Resnick (dir.), Knowing, Learning, and Instruction: Essays in Honor of Robert Glaser (p. 361-392). Hillsdale, NJ: Lauwrence Erlbaum.

Bereiter, C. et Scardamalia, M. (2003). Learning to work creatively with knowledge. In E. De Corte, L. Verschaffel, N. Entwistle et J. van Merriënboer (dir.), Powerful Learning Environments: Unravelling Basic Components and Dimensions (p. 73-78). Oxford, Angleterre: Elsevier Sciences.

Bereiter, C. et Scardamalia, M. (2010). Can children really create knowledge? Canadian Journal of Learning and Technology/La revue canadienne de l'apprentissage et de la technologie, 36(1), 1-24.

Brown, A.L. (1994). The advancement of learning. Educational Researcher, 23(8), 4-12.

Brown, A.L., et Campione, J. C. (1994). Guided Discovery in a Community of Learners. Boston, MA: The MIT Press.

Brown, A.L., Campione, J.C., Reeve, R.A., Ferrara, R.A. et Palincsar, A.S. (1991). Interactive learning and individual understanding: The case of reading and mathematics. In L.T. Landsmann (dir.), Culture, Schooling and Psychological Development (p. 136-170). Hillsdale, NJ: Lawrence Erlbaum Associates, Inc. Brown,

Chen, B., Chuy, M., Resendes, M., Scardamalia, M. et Bereiter, C. (2011). Evaluation by grade 5 and 6 students of the promisingness of ideas in knowledge-building discourse. In H. Spada, G. Stahl, N. Miyake et N. Law (dir.), CSCL2011 Computer Supported Collaborative Learning: Connecting Research to Policy and Practice (Vol. II) (p. 571-575). Honk Kong, HK: ISLS.

Chen, B., Resendes, M., Chuy, M., Tarchi, C., Bereiter, C. et Scardamalia, M. (2012a). Identifying promising ideas in a knowledge-building discourse. QWERTY-Interdisciplinary Journal of Technology, Culture and Education, 6(2), 224-241. 
Chen, B., Scardamalia, M., Resendes, M., Chuy, M. et Bereiter, C. (2012b). Students' intuitive understanding of promisingness and promisingness judgments to facilitate knowledge advancement. In J. van Aalst, K. Thompson, M.J. Jacobson et P. Reimann (dir.), The Future of Learning: Proceedings of the 10th International Conference of the Learning Sciences (ICLS 2012) (Vol. I) (p. 111-118). Sydney, AU: ISLS.

Chen, B., Scardamalia, M. et Bereiter, C. (2015). Advancing knowledge building discourse through judgments of promising ideas. International Journal of Computer-Supported Collaborative Learning, 10(4), 345-366.

Chuy, M., Resendes, M. et Scardamalia, M. (2010). Ways of contributing to knowledge building dialogue in science. Communication par affiche présentée dans le cadre du Knowledge Building Summer Institute (KBSI), Ontario Institute for Studies in Education (OISE), Université de Toronto, Canada.

Collins, A. et Halverson, R. (2009). Rethinking Education in the Age of Technology: The Digital Revolution and the Schools. New York, NY: Teachers College Press.

Hmelo-Silver, C.E. et Barrows, H.S. (2008). Facilitating collaborative knowledge building. Cognition and Instruction, 26, 48-94.

Krippendorff, K. (2012). Content Analysis: An Introduction to its Methodology (3e éd.). Thousand Oak, CA: SAGE publications.

Laferrière, T. (2005). Les communautés d'apprenants en réseau au bénéfice de l'éducation. Encounters on Education, 6, 5-21.

Laferrière, T. et Breuleux, A. (2002). L'école éloignée en réseau: revue des cas et des écrits. CEFRIO et ministères de l'Éducation et des Régions, Québec.

Merriam, S.B. (1998). Qualitative Research and Case Study Applications in Education. Revised and Expanded from "Case Study Research in Education". San Francisco, CA: Jossey-Bass.

Paavola, S., Lipponen, L. et Hakkarainen, K. (2004). Models of innovative knowledge communities and three metaphors of learning. Review of Educational Research, 74(4), 557-576.

Papert, S. (2000). What's the big idea? Toward a pedagogy of idea power. IBM Systems Journal, 39(3), 720-729.

Partnership for skills for $21^{\text {st }}$ century. (2008). $21^{\text {st }}$ century skills: Education and Competitiveness (A resource and Policy Guide). Document téléaccessible à l'adresse <http://www.p21.org/storage/documents/21st_century_skills_education_and_ competitiveness_guide.pdf $>$.

Patton, M.Q. (2015). Qualitative Evaluation and Research Methods (4e éd.). Thousand Oak, CA: SAGE Publications.

Savoie-Zajc, L. (2003). L'entrevue semi-dirigée. In B. Gauthier (dir.). Recherche en sciences sociales: de la problématique à la collecte des données (4e éd., p.293-316). Ste-Foy, QC: PUQ. 
Scardamalia, M. (2002). Collective cognitive responsibility for the advancement of knowledge. In B. Smith (dir.), Liberal Education in a Knowledge Society (p. 67-98). Peru, IL: Open Court.

Scardamalia, M. (2004). CSILE/Knowledge Forum. In A. Kovalchick et K. Dawson (dir.), Educational Technology: An Encyclopedia (p.183-192). Santa Barbara, CA: ABC-CLIO.

Scardamalia, M., Bransford, J., Kozma, B., et Quellmalz, E. (2012). New Assessments and Environments for Knowledge Building. In P. Griffin, B. McGaw et E. Care (dir.), Assessement and Teaching of $21^{\text {st }}$ Century Skills (p. 231-300). Dordrecht: Springer Netherlands.

Scardamalia, M. et Bereiter, C. (2003). Knowledge building. In J.W. Guthrie (dir.), Encyclopedia of Education. (2 éd.) (p. 1370-1373). New York, NY: Macmillan.

Yin, R.K. (2013). Case Study Research: Design and Methods (5e éd.). Thousand Oak, CA: SAGE Publications.

Zhang, J. et Sun, Y. (2008). Knowledge Building Beasures that Matter. Communication présentée dans le cadre du Knowledge Building Summer Institute, Ontario Institute for Studies in Education (OISE), Université de Toronto, Canada.

Zhang, J., Hong, H.Y., Scardamalia, M., Teo, C.L. et Morley, E. (2011). Sustaining knowledge building as a principle-based innovation at an elementary school. Journal of the Learning Sciences, 20(2), 262-307. 